\title{
Electrophysiological Evidence of Working Memory Updating Deficits in Children with Learning Disabilities*
}

\author{
Chunlei Liu \\ School of Educational Science \\ Qufu Normal University \\ Qufu, China
}

\author{
$\mathrm{Ru} \mathrm{Yao}$ \\ Dept. of Psychology and Special Education \\ National Institute of Education Sciences \\ Beijing, China
}

\begin{abstract}
Recent behavioral studies suggest that children with learning disabilities (LD) have difficulty in working memory (WM) updating. However, as behavioral performance represents the summed activity of multiple stages of processing, the temporal locus of the WM updating deficit remains unclear. Here, we used an event-related potential (ERP) paradigm to compare the temporal mechanisms of WM updating in 21 children with LD (aged between 10 and 12 years) and 21 agematched controls while they performed the running memory task. Behavioral results showed that children with $L D$ attained significantly lower accuracy when the task required updating. ERPs revealed that the occipital early $P 1$ component and parietal late positive complex (LPC) amplitudes were lower in children with LD compared to controls when the task required children to remember a new stimulus. The LPC (1000-1500) amplitude correlated with the performance of academic and updating behavior. These results provide a novel electrophysiological evidence that children with LD have a specific impairment in early visual attention processing and lately WM updating.
\end{abstract}

Keywords-learning disability (LD); working memory updating; running memory task; event-related potentials (ERP)

\section{INTRODUCTION}

Children with learning disability (LD) have some cognitive and neuropsychological processing difficulties associated with learning literacy and math. Recently, accumulating literature has identified working memory deficits as closely associated with specific LD, such as reading disability [1], mathematical disability [2], ADHD [3], or combined reading and mathematics disability [4][5]. So, some researchers have concluded that working memory may be a core deficit in children with LD, especially an impaired ability to update relevant information from working memory as a hallmark of the learning disorder because working memory (WM) updating is considered to be a key component of working memory [6]. WM updating is the ability to simultaneously integrate new relevant information and suppress data that is no longer relevant (Friedman et al., 2006).

This study was supported by Shandong Provincial Natural Science Foundation(ZR2015PC012), China.
Although there is converging evidence suggesting apparent links between LD and dysfunctional WMU processes, the temporal locus of the updating deficit remains unclear. Keage et al studied the ERP indices of WM updating in $\mathrm{AD} / \mathrm{HD}$, they have found delayed latency and attenuated amplitude of $\mathrm{N} 300$ and P450 component in the child with AD/HD during a one-back working memory task [7]. Recently, Horowitz-Kraus [8] found individuals with dyslexia displayed decreased ERP components (N100, P300) compared to skilled readers in the "target-locked" conditions of the Wisconsin Card Sorting Test. Therefore, studying these ERP components in relation with the running memory task in children with LD could highlight neuronal processes underlying the updating function in LD.

In the current study, we attempted to use ERPs to pinpoint the impaired cognitive abilities in the running memory task and explore potential neural markers of WM updating deficit in children with LD. This WM task requires not only a phonological loop, but also an effective updating function [9]. As the number of required updates rises (as more digits are presented), the task becomes more difficult and requires a better updating function. In order to obtain a pure measure of updating ERP effects, a control task is generally required rule out phonological loop component. This is typically done to obtain difference waves, in which the waveforms observed during the control task are subtracted from those obtained during the experimental task.

ERP indices of the early discrimination (P1/N1), selection of material $(\mathrm{P} 2)$, retrieval of relevant memories (N400), and late positive complex (LPC) will be assessed. A correlation analysis on ERP components and behavioral data collected during the task was conducted to find an ERP index sensitive to the WM updating function of children with LD.

\section{METHODS}

\section{A. Participants}

Twenty-one (12 boys, 9 girls) children with LD and 21 (11 boys, 10 girls) typically developing children were recruited from a public elementary school in Beijing. None of the children in the study attended special schools. All 
participants were in 5 th $(\mathrm{n}=22$, age: $10.38 \pm 0.57$ years $)$ or 6 th grade $(n=20$, age: $11.25 \pm 0.55$ years $)$. All children with LD were tested with standardized math, reading, and spelling tests, treated and diagnosed by a recognized paraprofessional or in a specialized center.

\section{B. The Running Memory Task}

Participants performed the running memory task akin to that of Kiss et al [10], while the EEG was recorded. The task required subjects to process strings of items of unknown lengths (the length of which ranged from 3 to 9 digits), and then to remember the last 3 digits in those strings, regardless of how many total digits were presented in the string. Control tasks only required the temporary storage of items, without any need to update the presented information. Therefore, participants had to continuously update the contents of their WM. For example, if a fourth digit is presented (position 4), participants needed to delete the first digit of the 3 in their digit memory sets and add in the new one.

\section{ERP Recording and Analysis}

ERP recording and analysis were conducted using SCAN4.3 system. ERPs were selectively averaged according to groups, task types (updating and control tasks), and updating positions (position 3 as the non-update position and positions 4 through 6 as update positions). Then, we obtained difference waves by subtracting the ERP waveforms observed during the control task from the experimental waveforms of LD group and control group. For ERP components, three-factor repeated measure ANOVAs were conducted on participant group type (LD group and control group), update position type (update position and non-update position), and electrode position ( $\mathrm{FPz}, \mathrm{Fz}, \mathrm{Cz}, \mathrm{Pz}$ and $\mathrm{Oz}$ ).

\section{RESULTS}

\section{A. Behavioral Results}

Behavioral data of accuracy and response time on the task are presented in "Table I".

TABLE I. MEANS ACCURACY AND RESPONSE TIME OF LDS AND CONTROLS

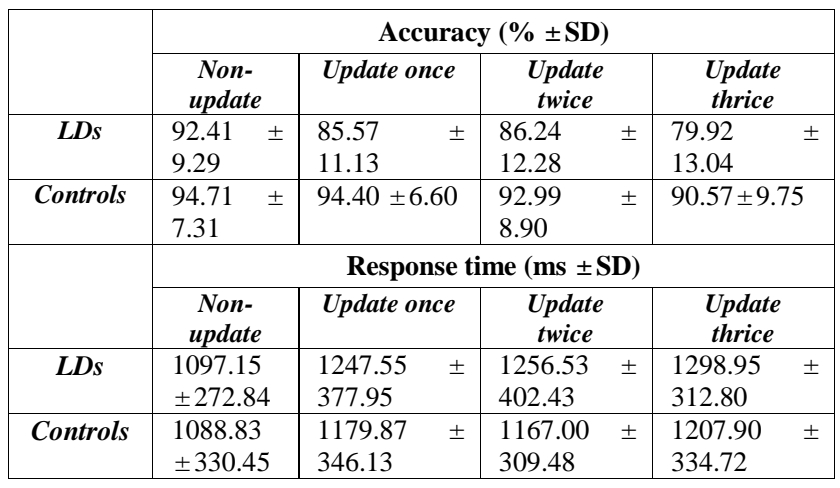

The LD children had significantly lower accuracy $\left(F_{(1,39)}\right.$ $\left.=7.85, p=0.008, \eta^{2}=0.17\right)$ compared with controls. There was a significant interaction between updating times and group $\left(F_{(1,117)}=2.99, p=0.03, \eta^{2}=0.07\right)$. Simple effect comparisons showed that there was no difference between the LD children and the control children under the nonupdate condition $(p=0.38)$. Under the update once, twice and thrice condition, the LD children's accuracy rate was significantly lower than that of the control children ( $p$ $s<0.05)$

In terms of response time, there was no significant interaction between updating condition and group $\left(F_{(1,117)}=\right.$ $\left.0.83, p=0.47, \eta^{2}=0.02\right)$. there was no significant main effect of group $\left(F_{(1,39)}=0.42, p=0.52, \eta^{2}=0.01\right)$. There was a main effect of updating times $\left(F_{(3,117)}=10.71, p<0.001, \eta^{2}=\right.$ $0.21)$. Post hoc comparisons showed that response time in the no-update condition was significantly shorter than in the update once, update twice, and update thrice conditions ( $p s$ $<0.01)$. There was no significant difference among the update once, update twice and update thrice conditions ( $p s>$ $0.05)$.

\section{B. ERP Results}

Grand-average ERP difference waves and topographical map were shown in "Fig. 1" and "Fig. 2".

- P1/N1. For P1 and N1 components within 100-140 $\mathrm{ms}$, result showed that interaction between update position type and electrode position was significant $(\mathrm{F}(4,140)=4.61, \mathrm{p}=0.004, \eta 2=0.11)$. Further analysis showed that $\mathrm{N} 1 \mathrm{in} \mathrm{Fz}$ was more positive for update position than non-update position, which is $\mathrm{N} 1$ 's positive position effect; $\mathrm{P} 1$ in $\mathrm{Oz}$ was more negative for update position than non-update position, which is P1's negative position effect. Comparison of position effect of two groups in different electrode sites showed us that P1's negative position effect in $\mathrm{Oz}$ was discovered in LD group, but not in control group.

- P2. For P2 within 165 to $215 \mathrm{~ms}$, result showed that interaction between update position type and electrode position was significant $(F(4,140)=3.525$, $p=0.025, \eta 2=0.089)$. Further analysis showed that in $\mathrm{Pz}, \mathrm{P} 2$ was more negative for update position than non-update position, which is $\mathrm{P} 2$ 's negative position effect. Comparison of position effect of two groups in different electrode sites showed us that $\mathrm{P} 2$ 's negative position effect was discovered in LD group, but not in control group.

- N400. Within the 300 to $500 \mathrm{~ms}$ time window of $\mathrm{N} 400$, the interaction between update position and electrodes was significant $(\mathrm{F}(4,140)=10.36, \mathrm{p}<$ $0.001, \eta 2=0.23)$. Simple effects analyses showed a positive position effect in $\mathrm{Fz}$, where ERP waveforms were more positive for the update position than the non-update position. $\mathrm{Pz}$ and $\mathrm{Oz}$ showed a negative position effect in which ERP waveforms were more negative for the update position than the non-update position. The positive position effect in $\mathrm{Fz}$ and negative position effect in $\mathrm{Pz}$ were observed in both groups. 


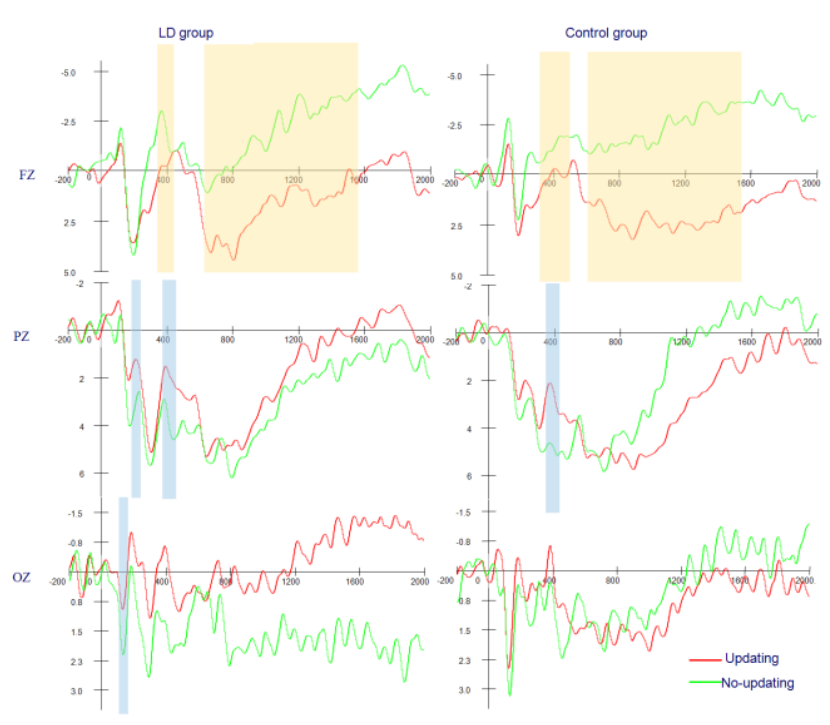

Fig. 1. ERP waveforms of LD children and control children time-locked to stimuli in update position and non-update position.

- LPC. Within the 500 to $1000 \mathrm{~ms}$ time window of LPC, the main effect of update position type was significant $(\mathrm{F}(1,35)=8.50, \mathrm{p}=0.006, \eta 2=0.20)$. ERP waveforms for the update position were more positive than those for the non-update position. The interaction between update position type and electrodes was significant $(\mathrm{F}(4,140)=13.50, \mathrm{p}<$ $0.001, \eta 2=0.28)$. Simple effects analyses showed that ERP waveforms in $\mathrm{FPz}, \mathrm{Fz}$, and $\mathrm{Cz}$ were more positive for the update position than the non-update position, indicating a positive position effect in anterior regions that was observed for both groups.

Within the 1000 to $1500 \mathrm{~ms}$ time window of LPC, the main effect of update position type was significant $(\mathrm{F}(1,35)$ $=25.30, \mathrm{p}<0.001, \eta 2=0.42)$, and ERP waveforms for the update position were more positive than for the non-update position. The interaction between update position type and electrodes was also significant $(\mathrm{F}(4,140)=17.75$, $\mathrm{p}<0.001$, $\eta 2=0.34)$. Simple effects analyses showed that ERP waveforms in $\mathrm{FPz}, \mathrm{Fz}$, and $\mathrm{Cz}$ were more positive for the update position than the non-update position. The interaction among participant group type, update position type, and electrodes was marginally significant $(\mathrm{F}(4,140)=2.62, \mathrm{p}=$ $0.069, \eta 2=0.07)$. Simple effects analyses showed that for the $\mathrm{LD}$ children, ERP waveforms in $\mathrm{FPz}, \mathrm{Fz}$, and $\mathrm{Cz}$ were more positive in the update position than the non-update position. ERP waveforms in $\mathrm{Oz}$ were more negative in the update position than the non-update position. For the control children, ERP waveforms in $\mathrm{FPz}, \mathrm{Fz}, \mathrm{Cz}$, and $\mathrm{Pz}$ were more positive for the update position than the non-update position.

\section{Correlations between ERP and Behavioral Data}

Within the 300 to $500 \mathrm{~ms}$ time window of N400, a significant positive correlation between the position effect at $\mathrm{Pz}$ and math score was observed for the LD children $(\mathrm{r}=$ $0.53, \mathrm{p}=0.019$ ), while the control children showed no such correlation. In the 1000 to $1500 \mathrm{~ms}$ time window of LPC, a positive correlation between the position effect at $\mathrm{Pz}$ and response time was significant for the control children $(\mathrm{r}=$ $0.58, \mathrm{p}=0.012$ ), while the LD children showed no such correlation.

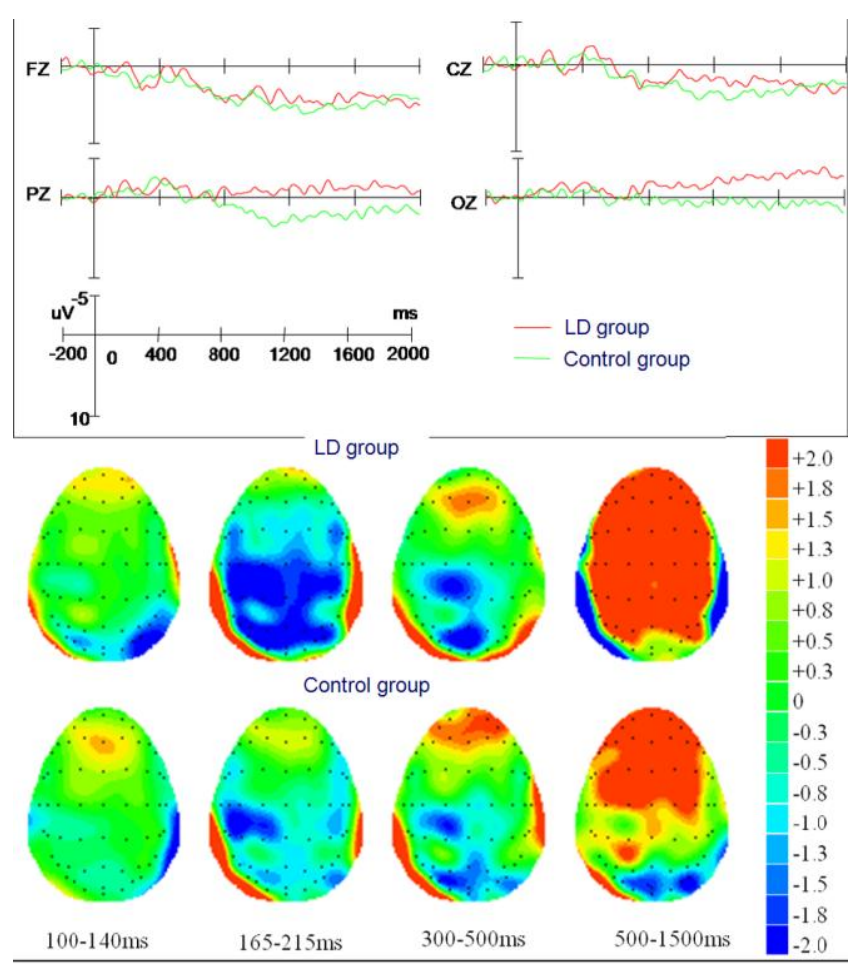

Fig. 2. Top: ERP difference waveforms of LD children and control children (update position minus non-update position). Bottom: topographical map of ERP difference waves (update position minus nonupdate position) of LD children and control children.

\section{CONCLUSION}

In the present study, we investigated behavioral and electrophysiological indices of WMU function in children with LD. The behavioral results revealed that children with LD performed more errors than typically developing children in the updating conditions. Since no updating function was involved in the zero update condition, participants only needed to store and remember the 3 digits presented, while an updating function is required when more than 3 digits are presented. Thus, our results indicate a specific difference in updating, but not storage, between the LD and controls. This is consistent with previous studies [10][11].

Our ERP results suggest that a negative position effect during the early stage of processing $(\mathrm{P} 1, \mathrm{P} 2)$, in which the LD children's waveforms over central parietal regions were more negative for update position than control children. The $\mathrm{P} 1$ is presumed to reflect early attention processing, the more a participant's attention is directed toward an upcoming target, the larger the amplitude of the P1 response to the target should be. Children with LD may have a passive attention toward an updating target. Our results also showed no difference in the ERPs within the 300- to 500-ms time window between the 2 groups. 
The scalp topography of the LPC is shown that differences exist between the two groups. After $500 \mathrm{~ms}$, the control children showed a positive position effect over anterior and posterior regions, while the LD children only showed a positive position effect over anterior and central regions, and a negative position effect over posterior regions.

Consistent with neurobiological basis of math (MD), reading (RD), and comorbid math and reading disability (MD+RD)[12], children with LD are characterized by limited abnormalities of LPC for memory updating involving posterior brain regions including parietotemporal and occipitotemporal area. Previous studies showed that the posterior parietal cortex maintains or shifts internal attention among the representations of items in WM [13]. Jonides et al [14] studies showed that the posterior parietal cortex plays not only a role in the storage but also in the proactive interference of verbal information in working memory. Therefore, typically developing children can shift between multiple representations of items, while children with LD showed deficits in representation shifting or proactive interference resolution. This could mean that the LD children performed worse than the control children at discarding the first digit in memory sets, suggesting that they may have difficulty in the suppression of old digits. Moreover, the LPC of children with LD is more located in anterior brain regions, which may be owing to the compensatory mechanisms of frontal brain areas in children with LD [12].

As for the relations among LPC, academic and behavior performance, some potential meaningful associations were found. In the 1000- to 1500-ms time window, the position effect at $\mathrm{Pz}$ and response time was significantly correlated for the control children but not the LD children. This suggests that a position effect within this time window may reflect functions related to information integration. Information integration is a comprehensive ability that is related to response time. It is a relatively predictive neurological index, which can be widely utilized research in diagnosis, assessment, and intervention training[15]. Within the 1000- to $1500-\mathrm{ms}$ time window, the control children showed a positive position effect over parietal regions, while the LD children showed a positive position effect only over prefrontal, frontal, and central regions. This could explain why the LD children did not show any such correlation.

There is converging evidence that early intervention might prevent or remedy the onset of LD [16][17]. Our results suggest that LPC might be as neurophysiological index of WM training-related changes in LD brain function and see if they were linked to clinical improvement.

To conclude, the present study provides evidence that children with LD have deficit in early attentional processes, they cannot recognize the new digit well. Furthermore, children with LD are characterized by limited abnormalities of late positive complex in posterior regions, which were related to suppressing the old digit and WM updating. Future studies with larger samples should account for the heterogeneity of LD by including subgroups in order to shed more light onto the neural correlates of the cognitive dysfunction in LD.

\section{REFERENCES}

[1] S. Wang, and S. E. Gathercole, "Working memory deficits in children with reading difficulties: Memory span and dual task coordination," $J$ Exp Child Psychol, vol. 115, pp. 188-197, May 2013.

[2] S. E. Gathercole, F. Woolgar, Calm Team, R. A. Kievit, D. Astle, T. Manly, and J. Holmes, "How common are wm deficits in children with difficulties in reading and mathematics?," J Appl Res Mem Cogn, vol. 5, pp. 384-394, Dec 2016.

[3] D. Stroux, A. Shushakova, A. J. Geburek-Hofer, P. Ohrmann, F. Rist, and A. Pedersen, "Deficient interference control during working memory updating in adults with adhd: An event-related potential study," Clin Neurophysiol, vol. 127, pp. 452-463, Jan 2016.

[4] F. De Weerdt, A. Desoete, and H. Roeyers, "Working memory in children with reading disabilities and/or mathematical disabilities," $J$ Learn Disabil, vol. 46, pp. 461-472, Sep-Oct 2013.

[5] P. Peng, and D. Fuchs, "A meta-analysis of working memory deficits in children with learning difficulties: Is there a difference between verbal domain and numerical domain?," J Learn Disabil, vol. 49, pp. 3-20, Jan-Feb 2016.

[6] B. Carretti, E. Borella, C. Cornoldi, and R. De Beni, "Role of working memory in explaining the performance of individuals with specific reading comprehension difficulties: A meta-analysis," Learn Individ Differ, vol. 19, pp. 246-251, Jun 2009

[7] H. A. Keage, C. R. Clark, D. F. Hermens, L. M. Williams, M. R. Kohn, S. Clarke, C. Lamb, D. Crewther, and E. Gordon, "Erp indices of working memory updating in ad/hd: Differential aspects of development, subtype, and medication," J Clin Neurophysiol, vol. 25 , pp. 32-41, Feb 2008.

[8] T. Horowitz-Kraus, "Pinpointing the deficit in executive functions in adolescents with dyslexia performing the wisconsin card sorting test: An erp study," J Learn Disabil, vol. 47, pp. 208-223, May-Jun 2014.

[9] N. Morris, and D.M. Jones, "Memory updating in working memory: The role of the central executive," Brit J Psychol, vol. 81, pp. 111$121,1990$.

[10] I. Kiss, S. Watter, J. J. Heisz, and J. M. Shedden, "Control processes in verbal working memory: An event-related potential study," Brain Res, vol. 1172, pp. 67-81, Oct 032007.

[11] F. Collette, M. Van der Linden, S. Laureys, F. Arigoni, G. Delfiore, C. Degueldre, A. Luxen, and E. Salmon, "Mapping the updating process: Common and specific brain activations across different versions of the running span task," Cortex, vol. 43, pp. 146-158, Jan 2007.

[12] S. Ashkenazi, J. M. Black, D. A. Abrams, F. Hoeft, and V. Menon, "Neurobiological underpinnings of math and reading learning disabilities," J Learn Disabil, vol. 46, pp. 549-569, Nov-Dec 2013.

[13] M. E. Berryhill, J. Chein, and I. R. Olson, "At the intersection of attention and memory: The mechanistic role of the posterior parietal lobe in working memory," Neuropsychologia, vol. 49, pp. 1306-1315, Apr 2011.

[14] J. Jonides, R. L. Lewis, D. E. Nee, C. A. Lustig, M. G. Berman, and K. S. Moore, "The mind and brain of short-term memory," Annu Rev Psychol, vol. 59, pp. 193-224, 2008.

[15] P. H. Leppanen, "Introduction to the special issue on brain eventrelated potentials as biomarkers of language and literacy development, feedback, and intervention," Dev Neuropsychol, vol. 38, pp. 507-513, 2013.

[16] J. C. Peijnenborgh, P. M. Hurks, A. P. Aldenkamp, J. S. Vles, and J. G. Hendriksen, "Efficacy of working memory training in children and adolescents with learning disabilities: A review study and metaanalysis," Neuropsychol Rehabil, vol. 26, pp. 645-672, Oct 2016.

[17] B. Opitz, J. A. Schneiders, C. M. Krick, and A. Mecklinger, "Selective transfer of visual working memory training on chinese character learning," Neuropsychologia, vol. 53, pp. 1-11, Jan 2014. 